\title{
Evaluation Studies of Motion Sickness Visually Induced by Stereoscopic Films
}

\author{
Yasuyuki Matsuura ${ }^{1,2}$, Hiroki Takada ${ }^{2, *}$ \\ ${ }^{1}$ Gifu City Women's College, Department of Cross-Cultural Studies, Gifu, 501-0192, Japan
}

${ }^{2}$ University of Fukui, Department of Human and Artificial Intelligent Systems, Graduate School of Engineering, Fukui, 910-8507, Japan

\begin{tabular}{l} 
A R T I C L E I N F O \\
\hline Article history: \\
Received: 21 January, 2021 \\
Accepted: 01 July, 2021 \\
Online: 03 August, 2021 \\
\hline Keywords: \\
3 D (3-dimantional) movie \\
Head Mounted Display (HMD) \\
Virtual Reality (VR) \\
Accommodation \\
Convergence \\
Visually Induced Motion Sickness \\
(VIMS) \\
Stabilometry \\
Biological signal
\end{tabular}

\section{Introduction}

In general, deviation occurs between the images formed on the two bilateral retinas when he/she gazes at a point with both eyes. This deviation is termed binocular parallax due to positional differences in the eyeballs. In humans, it plays an important role in perceiving three-dimensionality. Currently, most threedimensional (3D) movies and 3D television (3DTVs) use binocular parallax to distribute 2D images to both eyes to achieve stereopsis. Principle of the stereopsis using the abovementioned method is described in a book written by Euclid in approximately 280 B.C. [1, 2]. In the early half of the 19th century, Charles Wheatstone started the stereoscopic photography when he invested the binocular stereoscopic image display method "Stereoscope", which converted a pair of stereo images $[3,4]$. In recent years, various 3D video display systems such as mobile devices, free-

*Corresponding Author: Hiroki Takada, 3-9-1 Bunkyo Fukui, Fukui 910-8507,

Japan, +81-776-27-8795 \& takada@u-fukui.ac.jp

www.astesj.com

https://dx.doi.org/10.25046/aj060428 viewpoint TV, and 3D cinema have been developed. A few recent displays can also present binocular and multi-aspect autostereoscopic images although 3D glasses are generally required. In either case, however, there are the following issues.

(1) unpleasant symptoms including headache, vomiting, and eye strain.

(2) lack of ambience and realism.

Especially in Japanese 3DTVs, dynamic movements cannot be fully expressed since the binocular disparity is set to one degree or less (See section 3.3). Excessive measures against visuallyinduced motion sickness (VIMS) have been implemented without an appropriate manufacturing standard for stereoscopic video clips (VPs) and their display systems since the eye strain induced by 3D video viewing does not have been still elucidated.

\begin{abstract}
A B S T R A C T
mans have experienced motion sickness and possessed the knowledge of stereopsis since Euclid first recognized the concept of depth perception in human vision. Further, the motion sickness is including a sensation of wooziness and nausea that has been documented since approximately 400 B.C., when it was mentioned in the Aphorisms of Hippocrates. incleasant symptoms including visual fatigue. Despite the increased use of Inconsistency between convergence and lens accommodation is suspected as a cause of which motion sickness induced by stereoscopic viewing have not yet been identified. A indicated that the accommodative power did not correspond to the distance of convergence inconsistency and the unpleasant symptoms remains unclear. Therefore, we introduce relevant fields of science and technology.
\end{abstract}

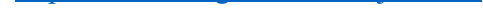


In $3 \mathrm{D}$ video viewing, it is generally understood that the lens is accommodated to the depth of the screen that displays the image, whereas the eyes converge at the position of the $3 \mathrm{D}$ object, which is a common idea of eye strain with $3 \mathrm{D}$ image viewing. As might be expected, the convergence and lens accommodation are consistent in natural vision. The discrepancy is considered to be cause of the eye strain and the motion sickness induced by stereoscopic viewing [5-6].

According to [7], if the viewing conditions are sufficiently bright, the depth-of-field (DOF) of a target has a mean difference in the order of 1.0 Diopter, and the accommodation-convergence conflict discussed above is a particular problem only in the case of proximity displays such as head-mounted displays (HMDs) and smart glasses [8]. Several methods are available to reduce motion sickness induced by the accommodation-convergence conflict, such as accommodation-invariant near-eye displays [9], light field [10], and rapid adjustment of the focal length to the congestion distance, "variable focus" [11, 12]. The first method displays images as if they are in focus even when the focal length and convergence distance are inconsistent. The second is a technology that generates images close to the visual sensation of the naked eye by taking photos and VPs from multiple viewpoints simultaneously. In addition, optical components in artificial lenses have been developed to change the focus of the human eye intentionally by changing the lens [13]. In addition, as a countermeasure that ignores the accommodation-convergence conflict, a notable method reduces the symptoms of motion sickness by providing the viewer with sound and vibration synchronized with the VPs [14].

Factors associated with the DOF include pupil diameter and resolution. Therefore, image viewing conditions definitely influence the pupil diameter. Most previous studies used high DOF to prevent blurriness, resulting in a measurement environment quite different from everyday conditions.

Moreover, the distribution of the convergence fusional limits in stereoscopic images was obtained in [15], where $84 \%$ subjects were able to see a stereoscopic image with a binocular disparity of two degrees. Notably, a single target without a surrounding image was used in the study. Generally, in absence of another parallax image, an accommodation-convergence process that merges double images into a single one functions as a positive feedback system [15].

Deviation between the eyeball positions causes differences in the formed retinal image because of the approximately $6 \mathrm{~cm}$ interpupillary distance between the bilateral eyes. The human ability to detect this difference in the retinal images between the bilateral eyes is nearly 10 times more accurate than normal visual acuity. When the deviation is too large, the image information from the bilateral eyes cannot be fused and a double image is formed, making the anteroposterior relationship unclear. A remarkable percentage of the population cannot perceive 3D vision by binocular parallax alone [16-17]. Stereopsis test methods include the T.S.T. (Titmus Stereo Test) and Lang (Lang Stereo Test).

However, it has been reported that there are some influence of stereoscopic viewing on health, which causes unpleasant symptoms, such as visual fatigue, headache, and the vertigo [1819]. Severity of VIMS is not affected only by construction of the images but also by the viewing environment. It has also been reported that prolonged viewing of stereoscopic displays can cause several health hazards such as severe visual fatigue and headaches [20-22]. On the side notes, it has been reported that the elderly with the mild cognitive impairment (MCI) tends to have a strong interest in stimulation by stereoscopic images [23]. Pregnant women, children, the elderly, and those who consumed alcohol should refrain from stereopsis in a few cases since it is easy for them to be influenced by the health problems associated with stereopsis [24]. However, it is necessary to indicate further hygienic investigations because of little knowledge of the biological effects such as visual fatigue and the VIMS [25].

This report provides an outline of the principle of stereopsis for various displays and the biological effects involved in the stereoscopic vision. Based on the forefront research in this field their clinical significance is also stressed for the description of future prospects.

\section{Stereopsis principle and presentation methods}

In humans, the two eyeballs are aligned approximately $6 \mathrm{~cm}$ apart horizontally. There are always subtly differences between two images formed on each retinas when a person sees an object with the bilateral eyes. Although the image formed on the retina is two-dimensional (2D), the brain reconstructs the information from the bilateral eyes and identifies the condition and the positional relationship of objects occupying the $3 \mathrm{D}$ space. Perception of the space and positional relationship is achieved through the eyeballs (lens accommodation and binocular convergence), difference in the eyeball position (binocular parallax and monocular movement parallax), and experience (sizes of objects, perspective, overlapping objects, texture, and shadows). These items are described below.

\subsection{Lens accommodation}

The mean diameter of the eyeballs in adults is about $22-25 \mathrm{~mm}$ and the weight is about 6-8 g. The cornea, anterior chamber, and lens are present in the anterior region and refract light coming into the eye to form images on the retina in the posterior region of the eyeball. However, the eyes cannot simultaneously set the focus on near and distant objects, and the focus has to be adjusted corresponding to the distance of each object. This is termed lens accommodation in the ocular optical system. In humans, the lens is accommodated by changing the lens thickness and curvature, adjusting the focus.

The annular ciliary body is present around the lens. Zinn's zonule radiating from the lens connects to the ciliary body. The ciliary muscle contracts in near vision, and relaxation of Zinn's zonule thickens the lens, shortening the focal distance and forming an image on the retina. In distance vision, the ciliary muscle is relaxed and Zinn's zonule extends the lens radially, which thins the lens and lengthens the focal distance, forming an image on the retina.

The range of lens accommodation is limited, and the limits of near and distance visions are termed near and far points of accommodation, respectively. However, hyperopia or myopia markedly varies among individuals and with aging. The ranges of these represent the accommodation ability, and the accommodation range is wide in young people. Changes in 
accommodation ability with aging are mainly due to shifting the near point of accommodation to a distant site because the lens loses elasticity with aging and become unable to readily increase the lens thickness.

\subsection{Convergence (binocular convergent movement)}

Our vision can perceive the target in detail within a narrow range of only about $1-2^{\circ}$, termed the central fovea. The central fovea contributes to vision in a high-definition field. To see an object, the visual axes of the bilateral eyes have to be set toward the object. The angle formed by these visual axes of the bilateral eyes is the convergence angle, and the distance to the intersection of the visual axes is termed the convergence distance (Figure 1). Convergence (binocular convergent movement) represents horizontal eyeball movement by simultaneous inward rotation of the bilateral eyes to gaze at an object and form an image in the central fovea on the retina, and convergent movement causes 'cross-eyes'[26]. Lens accommodation also has to change corresponding to the distance of the object. The voluntary muscles (ocular muscles) responsible for eyeball movement are roughly divided into the extra- and intra-ocular muscles, and a factor of the extraocular muscle, convergence, and that of the intraocular muscle, accommodation, are useful clues for the visual system to perceive distance [27-28]. Convergent movement occurs in response to the clue of depth direction, and deviation (parallax) of the image on the retina between the bilateral eyes is the typical stimulation. It is an eyeball movement occurring almost simultaneously with lens accommodation to enable 'seeing' in the depth direction in a 3-dimensional space [29]. Therefore, convergence is not conjugated movement of the bilateral eyeballs unlike optokinetic nystagmus which occurs to fix images formed on the retina and vestibulo-ocular reflex which maintains and stabilizes images on the retina even in the presence of body movement [29].

The visual system is capable of identifying the conditions of convergence and accommodation using an efferent copy, which is the command from the brain to the ocular muscles, or proprioceptor signals of the ocular muscles or both information [30]. Regarding the control system model of convergence and accommodation, there is a model in which the extra- and intraocular muscle are moved so as to fuse the images on the retina of the bilateral eyes regarding the distances of the target object of convergence and accommodation, as input signals, the focus (blurring) on the retina as 0 , and convergence and accommodation as output signals [31-32]. In the control system, crosslinks from the convergence control system to the accommodation control system or vice versa are present, and convergence and accommodation influence each other. Changes in convergence induced by changes in accommodation are termed accommodative convergence, and changes in accommodation induced by changes in convergence are termed convergence accommodation.

\subsection{Deviation between the eyeball positions}

In binocular stereoscopic images, which are currently mainstream, the viewer makes sense that the displayed object is present from the depth to the front side of the screen utilizing convergent movement and binocular parallax described above to show the image 3-dimensionally (Figure 2). Medial and lateral movements of the eyeballs are termed convergence and divergence, respectively.

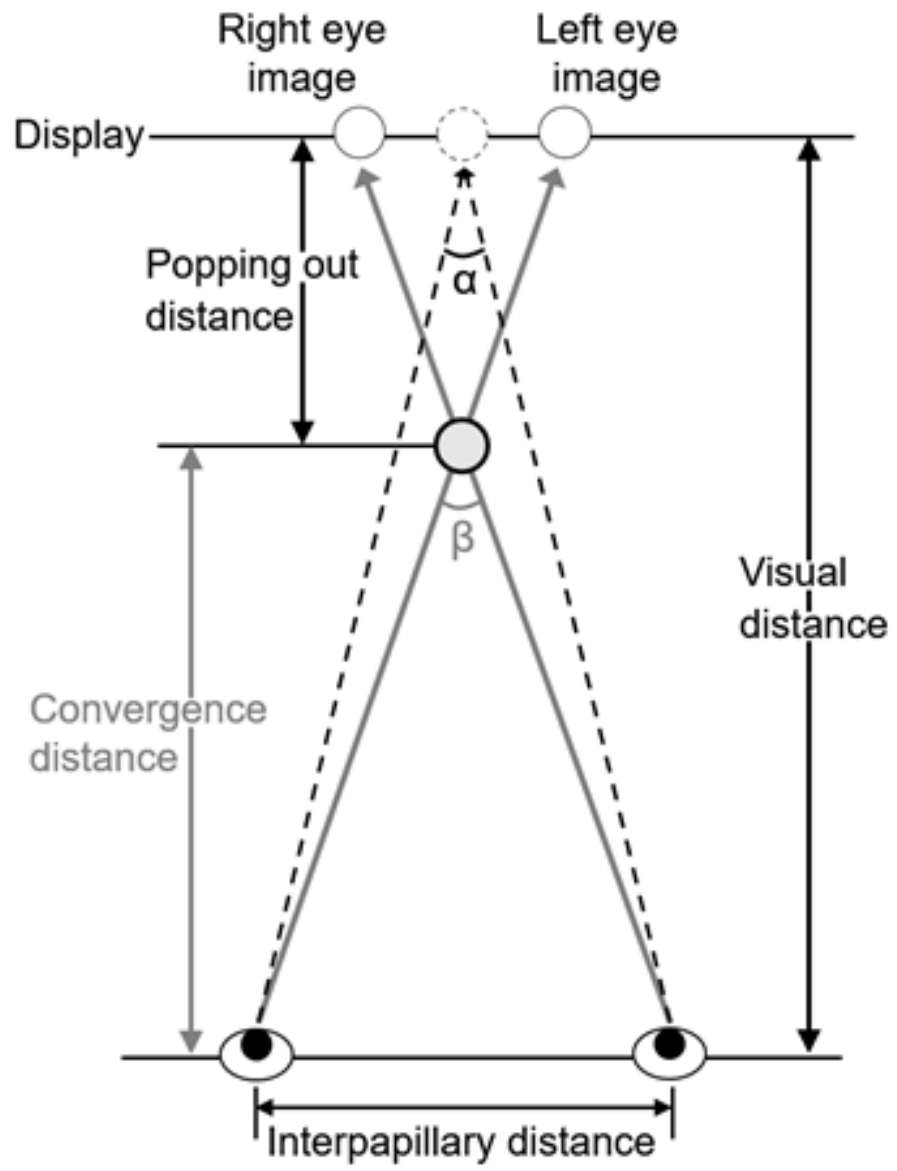

Figure 1: Eye convergence on seeing 3D display. $\alpha$ and $\beta-\alpha$ are defined as angle of convergence and parallactic angle, respectively.

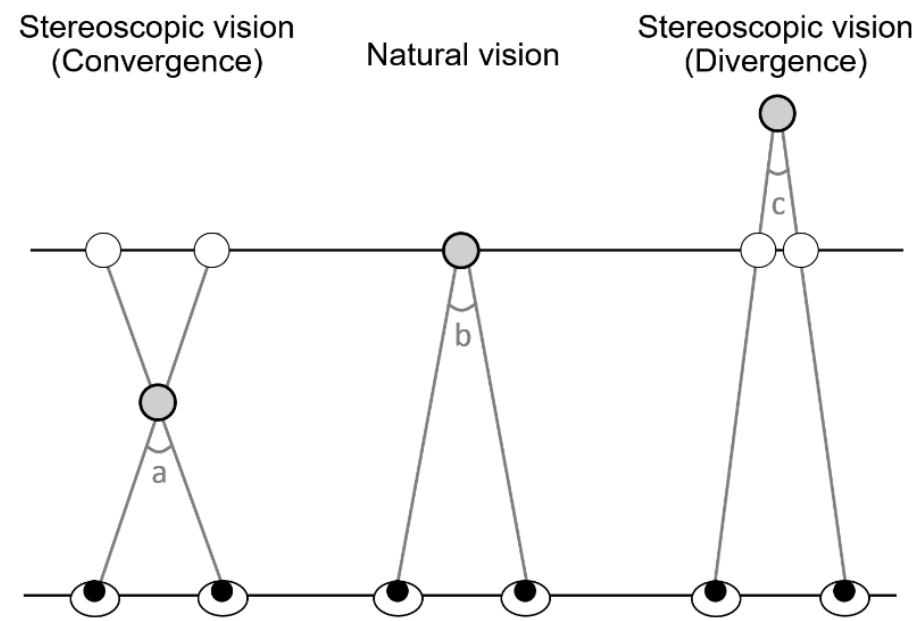

Figure 2: Binocular stereoscopic vision (Convergence and Divergence). a-b: Parallax angle while viewing an object/image in 3D popping out of the screen/display surface, c-b: that sinking in the surface.

Relative positions of the visual field and object change with movements of the object and observer, through which unevenness of the object and the anteroposterior relationship among several objects become perceptible. The direction and speed of visual target movement vary corresponding to the visual range and 
interpupillary distance. For example, when looking at the landscape from a car or train, nearby trees and signal masts move backward and distant mountains slowly move backward, but the very far sun and moon appear to move in the same direction as the observer.

\subsection{Experience}

Humans can perceive the depth and three-dimensionality of space even from a picture written on a flat surface. Various kinds of visual features bring about depth perception (perspective perception), depending on experience and other factors [33-34]. A visual object forming a large retinal image is perceived as present in the front and that forming a small retinal image is perceived as present in the back. When the retinal image size of an object is remembered by experience, the distance to the object is sensed [35]. In contrast, when the size is not known, it is unclear whether the distance is different or the size is different. When objects overlap, the object covering part of the other is perceived as present in front. It may be unclear whether the objects are overlapping or a hole is open based on the presence of overlapping objects alone, but when shadows produced by an illumination light are added, the anteroposterior relationship between objects can be identified.

The presence of shadows also produces three-dimensionality. In daily life space, the shape is convex when the shadow is formed below, and concave when the shadow is formed above. Feelings of unevenness and the anteroposterior relationship may be reversed depending on the shadow position. High and low contrasts induce a feeling of forward and backward movements, respectively. Similarly, light and dark induce a feeling of forward movement and depth of backward movement, respectively.

Regarding gradients of density difference and texture, perspective and inclined planes are perceived corresponding to differences in the density among elements constituting texture and the state of change (gradient). The appearance of the size decreases with an increase in the distance from the observer, and the distance between objects decreases by $1 / 2$ squared. Thus, it is easier to sense depth in the presence of a regular arrangement. This clue is closely related to perspective.

Perspective includes linear and aerial perspectives. In linear perspective, the feeling of depth is produced by composition on the assumption of a viewpoint position and distant vanishing point, and this is applied to perspective [36]. The Last Supper by Leonardo da Vinci is a typical example drawn by perspective. This work employed a one-point perspective setting the vanishing point in almost the center of the screen, which concentrates the line of sight to the theme and produces a sensation that a space extends in the back of the screen. In aerial perspective, near visual objects are clearly perceived while distant visual objects are dimly perceived, causing a feeling of depth. In real life, distant objects are blurred due to light scattering by air, giving a sense of perspective. When the size and shape are the same, clearly seen objects are felt near, and blurred objects are felt to be present at the back [37-38].

\subsection{Stereoscopic image display technique}

Stereoscopic image display methods include eye glass-type and naked-eye binocular stereoscopic display systems, multiview and depth information presentation systems, and wavefront reconstruction and space image systems. Of these, the binocular stereoscopic display system utilizes binocular parallax, in which 2 images with parallax in the horizontal direction are individually presented to the bilateral eyes, and it is generally used as a stereoscopic image display method [3, 39-40].

The image presentation methods employing the binocular stereoscopic display system include the side-by-side method in which the bilateral images are horizontally arranged, top-andbottom method in which the bilateral images are vertically arranged (images for the left and right eyes are set at top and bottom, respectively), the line-by-line method in which the bilateral images are arranged alternately on each line horizontally or vertically, and Power 3D method [41-42].

\section{Biological effects of image viewing}

Regarding the biological effects of stereoscopic viewing, adding camera shake-like vibration to the entire image and dynamic changes that involve computer graphic images to induce a feeling of being present at the place are likely to cause VIMS. Stimulation with stereoscopic images induces 3D sickness whose symptoms are similar to motion sickness. This is not limited to stereoscopic images, and viewing images and rapidly moving screen that requires blinking may cause headache, vomiting, and vertigo. These and other similar events can be broadly defined as VIMS.

\subsection{Biological effects of motion sickness induced by stereoscopic viewing}

Motion sickness is including a sensation of wooziness and nausea known since approximately 400 B.C. In [43] , the author described that "When Hellebore has been taken, let the body be general kept in motion, enjoying less rest, and less sleep. For even sailing proves that motion disturbs the functions of the body." In [44], it is written that "they felt sick due to vehicle sickness in a Japanese oxcart (Figure 3) and all appeared inverted to their blinking eyes." in the first half of the 12th century. Currently, besides vehicle motion sickness and VIMS, space sickness has also been reported [45-46]. As the space motion sickness symptoms develop in zero gravity, vomiting in a space suit helmet during extravehicular activity may cause suffocation. People have suffered the unpleasant symptom of motion sickness for long.

From the viewpoint of preventive medicine, it is important to accumulate the basic studies on stereoscopic viewing because stereoscopic viewing involves both positive [47] and negative aspects. Contrary to what you might think, there are a few reports on the former. Firstly, cases demonstrating the effect of antisuppression exercise of intermittent exotropia and the pleoptics have been reported by using stereoscopic image techniques [48]. In [47], the authors reported that "the accommodation training using 3D movie had temporarily improved visual acuity and seemed to lead to a decrease in asthenopia in their experiment."

Unpleasant symptoms such as visual fatigue, vomiting, and headache are often caused by stereoscopic images utilizing binocular stereopsis, which depends on the viewing conditions [49]. Integrating several sensations, such as those from the skin and somatic sources, the body perceives space. Since the space perception excessively depends on the visual function, visual sensation among others carries the major burden while viewing stereoscopic ones. In most cases after viewing those images, the 
symptoms associated with the motion sickness disappear when you stop watching, however, it may last almost a day in severe cases of the VIMS, which is not caused only by images but also by simulators. Sensations other than the visual one can be given by the simulators in which there is deviation between their sensations and motions included in the video film. In the simulator sickness, the motion sickness is also amplified by flickering in the screen. Furthermore, it has been reported about ataxia in the simulator sickness. and the US navy prohibits the persons with experience of the simulator sickness from boarding within 24 hours after simulator operation [50]. Thus, these kinds of the knowledge may give a key consideration of the motion sickness including the car sickness.

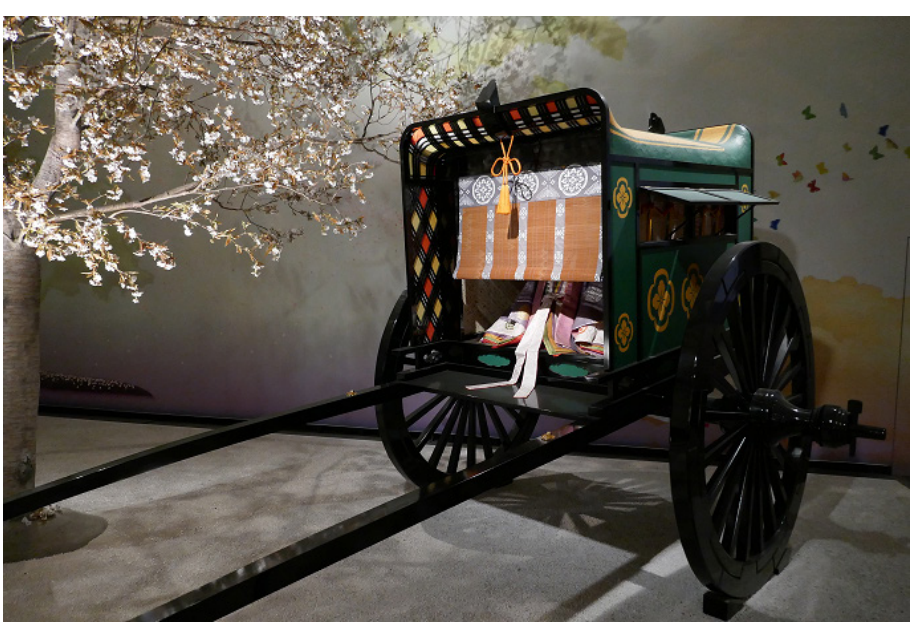

Figure 3: Japanese oxcart

The VIMS is influenced by auditory [51], visual [52-53], olfactory [54], deep [55], and other sensations. The followings are case studies. A worker operating the remote control of a large power shovel has developed severe ophthalmalgia and headache one month after starting work owing to stereoscopic image viewing, and his quality of life subsequently deteriorated [56]. In addition, there is another report that a boy has developed acute internal strabismus due to 3D movie viewing [44].

Early research considered the overstimulation theory for explaining the mechanism behind the onset of motion sickness. According to this theory, the acceleration of a vehicle causes the overstimulation of the visceral and vestibular organs, leading to an excitement of the hypothalamus, which induces the vestibulovegetative reflex, causing various symptoms of the motion sickness. Instead of this overstimulation theory, it is necessary to develop some mechanism of the motion sickness because the motion sickness was found even in microgravity environments. According to the sensory conflict theory $[18,57]$, actual sensory information such as visual, vestibular, and somatosensory one is compared with that of the episodic memory in the central nervous system. The motion sickness would be induced only if a sensory information combination were different from that expected from the memory [58]. The vestibular stimulation is transmitted to the vomiting center in the medulla oblongata via the vestibulovegetative system. The vestibular and autonomous nerve systems are anatomically and electrophysiologically closely connected, strongly suggesting their relationship with the unpleasant symptoms of motion sickness [59]. When a motion sickness- inducing rotational load is provided to rats, it increases the histamine level in the brainstem and hypothalamus, which is related to vomiting during the motion sickness [60].

Severity of the motion sickness can be quantitatively evaluated in accordance with the analysis of the body sway, which is regarded as an output of the equilibrium system. In general, it is difficult to obtain significant difference from statokinesigrams and/or their area of sway, sway values, total length, and total locus length per unit area, with their eyes open because the visual information helps subjects to keep upright posture. In recent decades, numerical analysis of the mathematical model of the body sway shows the possibility to find significant differences with eyes open (See section 3.3) [61].

In case of movies and 3DTV utilizing systems to display binocular stereoscopic images, the biological effect of stereoscopic viewing cannot be ignored. Figure 1 shows binocular parallax determined by the positional relationship between bilateral eyes and the object in stereoscopic images, which depends on the distance between the centers of your two eyes (interpupillary distance: PD). Using binocular parallax, stereopsis is realized by individually distributing 2D images to observers' left and right eyes. It also depends on the viewing conditions (viewing position, darkness and light in the room, and physical conditions). Therefore, certain biological effects occurring during stereoscopic viewing can be attributed to individual differences in viewing conditions, interpupillary distance, and visual function of the viewers. Studies on these differences have been reported $[56,62]$.

\subsection{Causes of motion sickness and visual fatigue by stereoscopic images}

According to the investigation of the relationship between individual differences in visual function and parallax range of stereoscopic images, persons with high accommodation convergence have a narrow range of comfortable visual field, where he/she is prevented from suffering discomfort of popping out stereoscopic images [63]. A positive correlation has been found between the grade of phoria and subjective evaluation for degree of stereoscopic viewing-induced fatigue [64]. As the side notes, there is less visual fatigue in persons with slight exophoria while viewing stereoscopic images [65]. Hence, considering individual differences in visual function is necessary while discussing the biological effects of stereoscopic viewing.

Theories of "inconsistency between convergence and accommodation" and the "influence of excess parallax" have been suggested in order to explain other causes for stereoscopic viewing-induced fatigue besides differences in the individual visual function. The former states that the visual fatigue would be caused by inconsistency between the accommodation and convergence distances, which also increases the accommodation load. The later states that the binocular parallax increased to emphasize the stereoscopic effects.

The following is frequently described as an effect of the binocular stereoscopic display system upon our body; accommodation and convergence contradict each other during stereoscopic viewing because we just focus upon the surface of the display and simultaneously adjust the convergence to the stereoscopic object popping out from it [66, 67]. Most of 
researchers believe that there is deviation between the accommodation and the convergence during stereoscopic vision, however, the authors reported that the accommodation was not fixed to the surface of the display during stereoscopic vision [68].

The range in which the anteroposterior regions of the focused region appear to be in focus is termed DOF, which is generally approximately $\pm 0.2-0.3$ Diopter in humans $[69,70]$. We would not feel inconsistency between the accommodation and the convergence when a stereoscopic object is presented within the DOF [71]. This also resolved the inconsistency problem during stereoscopic viewing, inhibiting the unpleasant feeling observed in a few studies [31, 72], whereas fatigue was caused in presence of a temporary change of binocular parallax, although the change was within the DOF in another study $[73,74]$.

A theory suggests that because the lens accommodation and convergence are simultaneously changing in opposite directions, their functions become unstable, and efforts to fuse the images created by the bilateral eyes are assumed to be the cause of fatigue [75-77]. In addition, we are conducting preliminary experiments on the illuminance of the experimental environment and interpreting the experimental data so far. The pupil diameter is affected when the environmental illuminance changes. When the illuminance is lowered, the pupil diameter becomes larger, and the DOF becomes shallower. We considered that it is easy to be induced the motion sickness because there is a discrepancy between the lens accommodation and the surface of the display, which is not included in the range of the DOF [78].

\subsection{The current trend of motion sickness and visual fatigue by stereoscopic images}

Because both the convergence and lens accommodation fit the objects in natural stereopsis, they do not contradict each other. The following hypothesis as described in section 3.2 seems to have captured the hearts of many 3D image engineers and researchers, i.e. it is often considered that their inconsistency is the cause of the VIMS. In 2011, 3D Consortium (3DC) formulated safety guidelines in which the range of comfortable parallax angle for stereoscopic images was specified as $\pm 1.0^{\circ}$ [79-81]. This is also based on the hypothesis and studies serving as the basis for this comfortable parallax range reported by [79] and [80]. Setting the change in binocular parallax within approximately $1^{\circ}$, approximately $87 \%$ subjects could fuse and observe stereoscopic images under the experimental conditions of their studies [79-81]. After these reports were published, however, this finding about the fusion limit was refuted as follows.

It was reported that stereoscopic images at a parallax angle up to approximately $2^{\circ}$ could be fused and observed by approximately $84 \%$ subjects [15]. It was concluded that the difference in the binocular parallax between [15] and [80] was due to premature processing deviating from elementary statistics. In recent decades, it has been discussed it is appropriate to revise the comfortable parallax range for stereoscopic images to $2^{\circ}$ [82]. As observed during natural viewing, the convergence and lens accommodation in the young were synchronized in accordance with their observation. No inconsistency was observed while viewing stereoscopic video clips under medium illuminance [83].
It is important for researchers in this field to set the visual environment, which can affect the results of the experiment. Thus, the experimental results of [83] cannot be simply compared with those of previous studies because the environment of [83] is different from those of experiments under low illuminance in the previous studies. In addition, the stereoscopic images were drawn by using "power 3D method" [41]. In [25], the author states that "no study has clearly shown the association although it is generally assumed that long-term stereoscopic viewing leads to unpleasant feelings including the visual fatigue." Several points presently remain unclear with regard to the accommodation-convergence mismatch during stereoscopic viewing and the discomforts.

\section{Mathematical models of the body sway to evaluate the motion sickness}

Severity of motion sickness is measured by stabilometry in accordance with the consideration of equilibrium function [78, 84]. In stabilometry, recording of stabilograms for 60 seconds begins when the standing posture stabilizes. Statokinesigrams are composed of each component of the stabilograms. Indices such as area of sway, total locus length, and total locus length per unit area are classically estimated to analyze statokinesigrams [84-85]. The latter is known as a parameter of the fine control of standing posture by the proprioceptive reflexes [86]. In addition to the abovementioned indices, an index termed sparse density was proposed in consideration of the non-linearity of the system to control upright position [87-88]. The following nonlinear system

$$
\begin{aligned}
& \frac{\partial x}{d t}=-\frac{\partial}{\partial x} U_{x}(x)+\mu_{x} w_{x}(t), \\
& \frac{\partial y}{d t}=-\frac{\partial}{\partial y} U_{y}(y)+\mu_{y} w_{y}(t)
\end{aligned}
$$

has been proposed for the description of the body sway where $w_{x}(t)$ and $U_{x}(x)$ represent the white noise and the time-averaged potential function (TAPF) in the lateral component, respectively. In general, the first term on the right-hand side is regarded as a linear function for each component [89-91], i.e. the body sway has been described as a Brownian motion [92-93]. Based on [94] and [95], the lateral component of the SDE (1.1) is assumed to be independent of the anterior-posterior component (1.2). Also, we did not obtain remarkable significance in the cross correlation between those components from the stabilograms measured in our experiments [87].

\section{Mathematical approach for the evaluation of body balance function}

\subsection{Improved deductive theory}

It is difficult to describe the abnormality in the body sway during the alcoholic load [87-88, 96] or the motion sickness. Prolonged exposure to a stereoscopic video clip, the mathematical model (1) has been investigated [97], but interaction between anteroposterior and lateral components cannot be neglected, and a new mathematical model is being investigated.

Non-linear stochastic differential equations (SDEs) (1) were obtained from our deductive theory [61, 78, 84]. The Markov process without abnormal diffusion is required by the randomness in the body sway, which is based on our observation. In most cases, 
of vibration load-induced motion sickness that the difference in stance width during upright makes a difference in the incidence of motion sickness [105].

$\mu_{x} \neq \mu_{y} \neq 1$ as to compare variations with the others in polygraphs that are measured and recorded in the experiments of the electrophysiology $[61,78,84]$. The TAPFs can be estimated as

$$
\begin{aligned}
& U_{x}(x)=-\frac{\mu_{x}^{2}}{2} \ln G_{x}(x)+\text { const., } \\
& U_{y}(y)=-\frac{\mu_{y}^{2}}{2} \ln G_{y}(y)+\text { const., }
\end{aligned}
$$

where $G_{x}(x), G_{y}(y)$, are expressed as distributions for each direction. Several minimum values of the TAPFs are often obtained from the stabilometry. In numerical simulations, local stability is also seen as motions with high-frequency near the minimal potential surface, where a high density of representation points is expected be generated by the SDEs. Inversely, degree of the local stability cannot be measured only by the total locus length per unit area, but also by the sparse density including more local information in the measurement [87].

\subsection{Application of our deductive theory}

The mathematical method in this chapter has already been applied to quantitate severity of the motion sickness induced by stereoscopic viewing and the blur of liquid crystal [94, 97-99]. Especially in [94], it has been discussed that peripheral vision contributed to an increase in the sway value with eyes closed after the exposure to a stereoscopic video clip. However, upright posture is stable with eyes open, in fact, the sway value is so small that the instability has been able to be evaluated while viewing video clips. As mentioned above, our deductive theory has been recently improved to enable comparison of variations among independent components. We have also succeeded in enhancing the accuracy of the evaluation during the exposure to stereoscopic video clips. In addition to the skewness, kurtosis, and standard deviation of the probability density distribution of the observed data, the translation error in the nonlinear analysis was herein used as an evaluation index for the numerical analysis of SDEs [61]. As a result, we constructed a new theoretical system to obtain the SDEs describing the equilibrium system from the measurement data of each subject.

\section{Evaluation of stereoscopic image-induced motion sickness}

In addition to the physiological methods involving autonomic nerve activity, subjective psychological methods have been well developed to measure the influence of the VIMS on the body. Simulator Sickness Questionnaire (SSQ) is a best-known measurement to assess the VIMS including the simulator sickness, which comprises 16 effective subjective items extracted from 1,119 paired data on the Motion Sickness Questionnaire (MSQ) measured before and after experiencing a simulator by using factor analysis [100]. The VIMS is also assessed using physiological measurements such as body sway, blood pressure, respiratory rate, electrocardiography, electrogastrography, perspiration, resistance value of the skin, and number of eye blinks [101-104]. In a study using the SSQ score, it was also reported by the group complaining

\subsection{Effect of background vision on the equilibrium system}

In previous studies, compared with visual pursuit, higher sway values including, the area of sway, total locus length, total locus length per unit Area, and sparse density during the peripheral viewing of 3D images were observed [94]. Especially in case of backgrounds, the appearance of actual space that humans perceive and that of 3D VPs is different, which is considered a reason for the influence of peripheral vision on the equilibrium system. In this section, we verify whether 3D VPs viewed without backgrounds influences the equilibrium system, and develop a mathematical model.

The body sway was measured during 1 min of video viewing, and thereafter, $3 \mathrm{~min}$ of standing with eyes closed after the prerest. Before and after this stabilometry examination, we performed a subjective evaluation of motion sickness symptoms using the SSQ. The smart glass MOVERIO BT-200 (EPSON, Nagano) was used to view the VPs used in the experiment of [106]. This device facilitates augmented reality (AR); however, to remove any external stimuli in the experiment other than those provided by the videos, they were projected on a black screen for measurement. In the video, spheres were fixed at four corners while another sphere moved through the screen in a complex manner.

We performed a two-way analysis of variance (ANOVA) that uses the persistence of the influence of exposure to VPs as a factor for each analytical index calculated from a statokinesigram. In the ANOVA results, several non-interacting main effects were observed for each pair of factors (solidity/backgrounds). According to the statistical analysis of the total locus length per unit area, there were main effects (1) on solidity when viewing VPs with backgrounds, and (2) of backgrounds when viewing 2D VPs. In this connection, backgrounds exerted a main effect when viewing 3D VPs in accordance with the ANOVA for sparse density S3. In addition, there was a main effect on solidity when viewing videos without backgrounds.

The equilibrium system was affected 1-2 min after viewing the 3D VPs with backgrounds. In addition, the SSQ result indicates that motion sickness may be caused by viewing 3D VPs with backgrounds. In addition, the sway values in the control were compared with those obtained after viewing 3D VPs without backgrounds (with their eyes closed). The area of sway and S3, both measured 2-3 min after the viewing, were significantly larger than those in the control. At that time, the total locus length per unit area was significantly smaller than that in the control. Subjects were allowed to use their peripheral vision; however, it was easy to focus on the central sphere, which was the same as the pursuit viewing of images because subjects viewed the VPs without backgrounds. Therefore, the influence reduction was observed 1$2 \mathrm{~min}$ after the $3 \mathrm{D}$ viewing. Moreover, $3 \mathrm{~min}$ after viewing, the instability of the system may increase owing to physical fatigue from maintaining an upright posture, leading to increased body sway. 
Table 1: Recent researches

\begin{tabular}{|c|c|c|c|c|}
\hline Authors & M. Malińska et al [89] & A. M. Baranowski et al [90] & T. H. Cho et al [91] & Y. Sawada et al [14] \\
\hline Year & 2015 & 2016 & 2017 & 2020 \\
\hline Apparatus & $\begin{array}{l}\text { 3D: Screen with Shutter } \\
\text { glasses } \\
\text { HMD: HMD with gloves }\end{array}$ & $\begin{array}{l}\text { Screen with 3D } \\
\text { glasses }\end{array}$ & $\begin{array}{l}\text { 3DTV with Polarization } \\
\text { glasses }\end{array}$ & $\begin{array}{l}\text { HMD when sitting on a } \\
\text { chassis of a scooter }\end{array}$ \\
\hline Experiments & $\begin{array}{l}\text { 3D: Watching part of } \\
\text { 'Avatar' } \\
\text { HMD: Training in } \\
\text { handling on the virtual } \\
\text { workstation }\end{array}$ & $\begin{array}{l}3 \text { genres (horror, action, and } \\
\text { documentary) with three } \\
\text { between-subjects viewing } \\
\text { conditions (director's 3D, } \\
\text { artificial 3D, and 2D) }\end{array}$ & 3D and 2D films & $\begin{array}{l}3 \mathrm{D} \text { video with Sound } \\
\text { and/or vibration riding } \\
\text { simulator motorcycle }\end{array}$ \\
\hline $\begin{array}{l}\text { Measuring } \\
\text { (Objective) }\end{array}$ & Electrocardiogram & & $\begin{array}{l}\text { Ocular parameters } \\
\text { (Accommodation, } \\
\text { Convergence, } \\
\text { Stereo-acuity, } \\
\text { Tear break-up time) } \\
\end{array}$ & \\
\hline $\begin{array}{l}\text { Measuring } \\
\text { (Subjective) }\end{array}$ & & $\begin{array}{l}\text { Fast Motion Sickness scale } \\
\text { (FMS) and } \\
\text { Self-Assessment Manikin } \\
\text { scale (SAM) }\end{array}$ & & FMS \\
\hline $\begin{array}{l}\text { Evaluation } \\
\text { methods }\end{array}$ & Statistical Analysis & Statistical Analysis & Statistical Analysis & Statistical Analysis \\
\hline
\end{tabular}

\subsection{Effects of duration on the equilibrium system}

In previous studies, it was shown that viewing 3D VPs affects body sway; however, there was no comment on the duration of viewing VPs. In this section, we examine the effects of duration on the body sway and introduce a mathematical model that describes the equilibrium system [107]. In addition, we succeeded in finding the temporal fluctuation in the mathematical model [108].

The experimental protocol of [107] used two patterns of measurements: following a standing pre-rest, 1 min with eyes open and $3 \mathrm{~min}$ with eyes closed; 2 min with eyes open and $3 \mathrm{~min}$ with eyes closed. A 3D VP with binocular disparity and a 2D VP for uniocular viewing were displayed on the $3 \mathrm{D}$ display $\mathrm{KDL}$ 40HX80R (SONY, Tokyo) installed $2 \mathrm{~m}$ from the subjects. The experiment considered the order effect, and we conducted the measurements for 2D and 3D VPs in random order. In addition, measurements for other durations were also performed on different days. Excluding the total locus length per unit area, we observed that the increase in sway values depended on the duration of the exposure to 2D VPs. In addition, sway values 1-2 min after viewing a 3D VP for 2 min were significantly greater than those while viewing it. Therefore, the influence of the viewing on the equilibrium system was seen even after the exposure to VPs. Regardless of the solidity of the VPs, the area of sway and the total locus length were significantly greater at 2-3 min after viewing than when viewing VP sway values. Similar results were observed in the control experiment. Therefore, in the experiment [107], an increase in the sway values at 2-3 min after viewing may not be caused by VIMS, but by fatigue in maintaining the upright posture. Hence, duration has an effect on the equilibrium function after viewing the VPs, and viewing the 3D VPs for 2 min continues to influence the equilibrium function for at least $2 \mathrm{~min}$ after viewing.

\section{Problems and future prospects}

Table 1 lists the recent research on this topic [14, 89-91]. The progression of TV technology to high image quality has facilitated the sales of naked-eye 3D displays for medical use and highdefinition glassless 3DTV and enhancement in the image quality of 3D images. Furthermore, recently there has been a rapid progression in weight reduction and enhancement in the performance of eyeglass-type wearable devices [109]. A small projector is attached to the frames of eye glasses and sun glasses, and images are projected either on the inner side of the lens, or projected in front of the eyes using a semitransparent binocular HMD with an integrated lens and projector or non- and semitransparent monocular displays. These new image display methods have already appeared, increasing the opportunities for stereoscopic viewing in various fields, for not only amusement but also medical care and industrial use.

The characteristic of our study introduced in section 4, 5 and 6 is that it not only aggregated experimental studies on the influence of stereoscopic viewing on visual function, but also helps establish scientific techniques to quantify motion sickness. Previous studies discussed inconsistency between convergence and accommodation without simultaneously measuring them; however, we performed stabilometry, simultaneous measurement of convergence and accommodation, and evaluated body balance and visual functions for a basic investigation of stereoscopic viewing-induced motion sickness in the experimental study. By increasing the number of subjects, performing close investigation 
with autonomic nerve evaluation using electrocardiography and electrogastrography, and evaluating the body balance function using body sway in a seated posture, this study facilitates safe stereoscopic viewing with less occurrence of VIMS.

Virtual Reality (VR) sickness can be caused by a visual illusion called vection [110-111] and movement on the screen while viewing $3 \mathrm{D}$ images. According to the sensory conflict theory, VR sickness can also be induced when passive movement creates a mismatch between information related to orientation and movement supplied by the visual and vestibular systems. This mismatch induces feelings of nausea. In particular, vection is easily caused in HMDs and on large-sized high-definition $4 \mathrm{~K} / 8 \mathrm{~K}$ displays. In addition, understanding of changes in the bio-signals during the vection helps us to confirm the previous studies [112]. It might improve our knowledge in the concept the motion sickness [112]. Therefore, it is important to examine the influence of vection on human bodies in detail.

\section{Conclusion}

This report provides an outline of the principle of stereopsis for various displays and the biological effects involved in the stereoscopic vision. Based on the forefront research in this field their clinical significance is also stressed for the description of future prospects. The spread of $3 \mathrm{D}$ and $4 \mathrm{~K} / 8 \mathrm{~K} \mathrm{TV}$ cannot progress unless the safety of stereoscopic images is secured, resulting in elimination from market competition. Therefore, prevention and alleviation of motion sickness stereoscopic viewing and providing basic documents to establish the safety criteria of 3D not only secure the extensive application of stereoscopic images and safety and relief of viewers but also contribute to technological development in Japan.

\section{Conflict of Interest}

The authors declare no conflict of interest.

\section{Acknowledgment}

This research has been supported in part by JSPS KAKENHI Grant Number JP17K00715, JP18K11417, and JP26870490.

\section{References}

[1] H. E. Burton, “The optics of Euclid," JOSA. 35(5), 357-372, 1945.

[2] I. P. Howard, B. J. Rogers, Binocular Vision and Stereopsis. New York: Oxford University Press, 1995.

[3] W. Charles, "Contributions to the Physiology of Vision. Part the First. On Some Remarkable, and Hitherto Unobserved, Phenomena of Binocular Vision," Philosophical Transactions of the Royal Society of London, 128, 371-394, 1838.

[4] B. David, The stereoscope; its history, theory, and construction, with its Application to the fine and useful arts and to education. London: John Murray, 1856.

[5] F. M. Toates, F. M. "Vergence eye movements," Documenta Ophthalmologica, 37(1), 153-214, 1974.

[6] H. G. Hoffman, D. R. Patterson, E. Seibel, M. Soltani, L. Jewett-Leahy, S. R. Sharar, "Virtual reality pain control during burn wound debridement in the hydrotank," Clin J Pain, 24, 299-304, 2008.

[7] R. Patterson, "Human factors of stereo displays: An update," Journal of the Society for Information Display, 17, 987-996, 2009.

[8] S. Nagata, "The binocular fusion of human vision on stereoscopic displays field of view and environment effects," Ergonomics. 39(11), 1273-1284, 1996.

[9] R. Konrad, N. Padmanaban, K. Molner, E. A. Cooper, G. Wetzstein, "Accommodation-invariant Computational Near-eye Displays. ACM
Transactions on Graphics,” 36(4), doi:10.1145/3072959.3073594, 2017.

[10] M. Broxton, J. Flynn, R. Overbeck, D. Erickson, P. Hedman, M. DuVall, J. Dourgarian, J. Busch, M. Whalen, P. Debevec, "Immersive light field video with a layered mesh representation," ACM Transactions on Graphics, 39(4), doi:10.1145/3386569.3392485, 2020.

[11] D. Dunn, C. Tippets, K. Torell, P. Kellnhofer, K. Akşit, P. Didyk, K. Myszkowski, D. Luebke, H. Fuchs, Wide Field Of View Varifocal Near-Eye Display Using See-Through Deformable Membrane Mirrors," IEEE Transactions on Visualization and Computer Graphics, 23(4), 1322-1331, 2017.

[12] K. Akşit, W. Lopes, J. Kim, P. Shirley, D. Patrick, "Near-eye varifocal augmented reality display using see-through screens," ACM Transactions on Graphics, 36(6), DOI:10.1145/3130800.3130892, 2017.

[13] W. Cui, L. Gao, "Optical mapping near-eye three-dimensional display with correct focus cues," Optics Letters, 42(13), 2475-2478, 2017.

[14] Y. Sawada, Y. Itaguchi, M. Hayashi, K. Aigou, T. Miyagi, M. Miki, T. Kimura, M. Miyazaki, "Effects of synchronised engine sound and vibration presentation on visually induced motion sickness," Scientific Reports. 10, DOI: 10.1038/s41598-020-64302-y, 2020.

[15] S. Nagata, "Distributions of "Vergence Fusional Stereoscopic Limit (VFSL)" of Disparity in Stereoscopic Display," Transactions of the Virtual Reality Society of Japan, 7(2), 239-246, 2002.

[16] W. Richards, "Stereopsis and stereoblindness," Experimental B rain Research, 10, $380-388,1970$.

[17] W. Richards, "Anomalous stereoscopic depth perception," JOSA, 61, 410419,1971

[18] J. T. Reason, J. J. Brand, Motion Sickness, London: Academic Press Inc, 1975.

[19] C. D. Balaban, J. D. Porter, "Neuroanatomic substrates for vestibuloautonomic interactions," J Vestib Res, 8, 7-16, 1998.

[20] K. Murata, S. Araki, K. Yokoyama, K. Yamashita, Y. Okumatsu, S. Sakoh, "Accumulation of VDT Work-Related Visual Fatigue Assessed by Visual Evoked Potential, near Point Distance and Critical Flicker Fusion," Ind Health. 34, 61-69, 1996.

[21] G. Hamagishi, "Ergonomics for 3D Displays and Their Standardization," The Technical Report of the Institute of Image Information and Television Engineers. 33(16), 9-12, 2009.

[22] K. Nishimura, T. Iwata, K. Murata, "Effects of 3-dimensional video games on visual nervous function," Akita J Med. 37, 85-91, 2010.

[23] T. Shibata, T. Kawai, K. Noro, A. Arimoto, T. Ohshima, S. Matsuoka, "A Study on the welfare application of stereoscopic 3D images for dementia patients," Ergonomics. 36(Suppl), 390-391, 2000.

[24] Samsung, Viewing TV using the 3D function http://www.samsung.com/ca/pdf/3D-tv-warning_en.pdf Accessed 30 November 2017.

[25] Ministry of Internal Affairs and Communications of Japan, "Final Report of Investigation Committee for 3D Television," 2012.

[26] M. Ikeda, What are your eyes looking at? Tokyo: Heibonsha, 1988.

[27] S. K. Fisher, K. J. Ciuffreda, "Accomodation and Apparent Distance," Perception, 17, 609-621, 1988.

[28] M. Mon-Williams, J. R. Tresilian, "Some Recent Study on the Extrarentinal Contribution to Distance perception," Perception, 28(2), 167-181, 1999.

[29] K. Uchikawa, K. Shinomori, Visual I: The faculty of sight and Initial function Tokyo: Asakurashoten; 2007.

[30] H. M. Burian, G. K. Von Noorden, Binocular Vision and Ocular Motility: Theory and Management of Strabismus St Louis: Mosby; 1980.

[31] N. Hiruma, T. Fukuda, "Viewing Conditions for Binocular Stereoscopic Images base on Accommodation Response," The IEICE transactions on information and systems D-2, 73(12), 2047-2054, 1990.

[32] C. M. Schor, "A Dyanmic Model of Cross-Coupling between Accomodation and Convergence: Simulations of Step and Freqency Responses," Optometry and Vision Science, 69, 258-269, 1992.

[33] H. Wallach, D. N. O'Connell, "The kinetic depth effect," Journal of Experimental Psychology," 45(4), 205-217, 1953.

[34] S. A. Linkenauger, M. Leyrer, H. H. Bülthoff, B. J. Mohler, "Welcome to wonderland: The influence of the size and shape of a virtual hand on the perceived size and shape of virtual objects," PloS one, 8(7), doi: 10.1371/journal.pone.0068594, 2013.

[35] G. Mother, Foundations of Perception, London: Taylor \& Francis, 2006.

[36] N. D. Cook, Harmony, Perspective and Triadic Cognition. New York: Cambridge University Press, 2011.

[37] S. Nagata, "Visual Sensitivities to Cues for Depth Perception," Journal of The Institute of Television Engineers of Japan, 31(8), 649-655, 1977.

[38] J. E. Cutting, P. M. Vishton, "Perceiving Layout and Knowing Distances: The integration, Relative Potency, and Contextual Use of Different 
Information About Depth, "Perception of Space and Motion, 22(5), 69-117, 1995.

[39] Y. Takaki, "Basics of Three-dimensional Displays," The Journal of The Institute of Image Information and Television Engineers, 67(11), 966-971, 2013.

[40] S. Aukstakalnis, Practical Augmented Reality: A Guide to the Technologies, Applications, and Human Factors for AR and VR, Boston: Addison-Wesley Professional, 2016.

[41] T. Fuji, M. Kosaka, T. Komuro, A, Shimotomai, "PC 3D Viewer Kit," Journal of The Society of Photographic Science and Technology of Japan, 72(4), 261-265, 2009.

[42] N. Fujiyoshi, M. Nagasawa, "Dawn of the Era of Three-dimensional Images," Mitsubishi Denki giho, 85(3), 2-6, 2011.

[43] Hippocrates, Coar T. The Aphorisms of Hippocrates with a translation into Latin, and English, London: Classics of Medicine Library, 1982.

[44] J. Ikegami, Konjaku Monogatari-Shu Honshobu (Last Part) Tokyo: Iwanamishoten, 2001.

[45] A. Graybiel, C. D. Wood, E. F. Miller, D. B. Cramer, "Diagnostic Criteria for Grading the Severity of Acute Motion Sickness," Aerospace Med, 39(5), 453-455, 1968.

[46] P. S. Cowings, K. H. Naifeh, W. B. Toscane, "The Stability of Individual Patterns of Autonomic Responses to Motion Sickness Stimulation," Aviat Space and Environ Med, 61, 399-405, 1990.

[47] A. Sugiura, M. Miyao, T. Yamamoto, H. Takada, "Effect of strategic accommodation training by wide stereoscopic movie presentation on myopic young people of visual acuity and asthenopia," Displays, 32(4), 219-224, 2011.

[48] T. Handa, "The present condition of three-dimensional films and utilization of three-dimensional technology in visual function tests and orthoptics procedures," Journal of Japanese Association of Certified Orthoptist, 41, 4552, 2012.

[49] H. Ujike, "Report of ISO International Workshop on Human Safety on Image," VISION. 17(2), 143-145, 2005

[50] US Navy. OPNAVIST 3710.7T, 2004.

[51] S. Takane, Y. Suzuki, T. Sone, H-Y. Kim, "A study on control of distance perception by simulation of HRTF," Proceedings of the Virtual Reality Society of Japan Annual Conference, 1, 55-58, 1996.

[52] T. Inoue, "Eye Movement and Accommodation when Viewing 2D and 3D Images," The Journal of the Institute of Television Engineers of Japan, 50-4, 423-428, 1996.

[53] R. S. Kennedy, K. S. Berbaum, W. P. Dunlap, L. J. Hettinger, "Developing Automated Methods to Quantify the Visual Stimulus for Cybersickness," Hum Factors Ergon Soc Annu Meet, 40-2,1126-1130, 1996.

[54] M. Ohsuga, T. Tatsuno, F. Shimono, K. Hirasawa, H. Oyama, H. Okamura, "Bedside Wellness - Development of a Virtual Forest Rehabilitation System," Stud Health Technol Inform, 50, 168-174, 1998.

[55] E. M. Kolasinski, Simulator sickness in virtual environments (ARI Technical Report 1027). Alexandria: U.S. Army Research Institute for the Behavioral and Social Sciences, 1995.

[56] H. Uchida, K. Hiwatashi, "Individual Difference of Stereoscopic Vision," Proceedings of the ITE Annual Convention, 29, 115-116, 1993.

[57] C. D. Balaban, J. D. Porter, "Neuroanatomic substrates for vestibulo-autonomic interactions," J Vestibular Research, 8, 7-16, 1998.

[58] K. Hirayanagi, "A present state and perspective of studies on motion sickness," The Japanese Journal of Ergonomics, 42(3), 200-211, 2006.

[59] N. H. Barmack, "Central vestibular system: vestibular nuclei and posterior cerebellum,” Brain Research Bulletin, 60, 511-541, 2003.

[60] N. Takeda, M. Morita, T. Kubo, A. Yamatodani, T. Watanabe, H. Wada, T. Matsunaga, "Histaminergic Mechanism of Motion Sickness Neurochemical and Neuropharmacological Studies in Rats," Acta Otolaryngologia, 101, 416-421, 1986.

[61] F. Kinoshita, H. Takada, "Numerical analysis of SDEs as a model for body sway while viewing 3D video clips," Mechatronic Systems and Control, 47(2), 98-105, 2019.

[62] M. Sato, "Individual Differences in Stereopsis," Journal of Japanese Society Opthaimpligical Optics, 35(2), 33-37, 2014.

[63] H. Mizushina, H. Ando, “The Relationship between Disparity Range for Comfortable Viewing of Stereoscopic Images and Individual Differences in Visual Function," The Technical Report of the Institute of Image Information and Television Engineers, 38(11), 23-26, 2014.

[64] T. Shibata, J. Kim, D. M. Hoffman, M. S. Banks, "The Zone of Comfort: Predicting Visual Discomfort with Stereo Displays,' J Vis, 11(8), 1-29, 2011.

[65] S. Kubota, K. Kudo, M. Takemoto, A. Shimada, Y. Nakamura, "Affect of Visual Acuity and Accommodation Speed on Visual Fatigue During Movie Viewing on 3D Television," The Journal of the Institute of Image
Information and Television Engineers, 67(7), J262-269, 2013

[66] F. M. Toates, "Vergence eye movements," Doc Ophthal, 37, 153-214, 1974.

[67] Ultra-Realistic Communications Forum, Ultra-experience Design and Evaluation Section, Working Group of Evaluation of 3D Images, "Report of Evaluation about Visual Fatigue of Stereoscopic Video Revised Edition," 2013.

[68] M. Miyao, S. Ishihara, S. Saito, T. Kondo, H. Sakakibara, H. Toyoshima, "Visual accommodation and subject performance during a stereographic object task using liquid crystal shutters," Ergonomics, 39(11), 1294-1309, 1996.

[69] F. W. Cambell, “The Depth of Field of the Human Eye," Journal of Modern Optics, 29, 157-164, 1957.

[70] W. N. Charman, H. Whitefoot, "Pupil Diameter and Depth-of-Field of Human Eye as Measured by Laser Speckle," Optica Acta, 24, 1211-1216, 1977.

[71] T. Kawai, H. Morikawa, K. Ohta, N. Abe, Basic Principles and Production Technology of Stereoscopic Images. Tokyo: Ohmsha, 2010.

[72] Y. Nojiri, H. Yamanoue, A. Hanazato, F. Okano, "Measurement of Parallax Dis tribution and its Applacation to the Analysis Visual Comfort for Stereoscopic HDTV," Proc SPIE. 5006, 195-205, 1993.

[73] S. Yano, M. Emoto, T. Mitsuhashi, "Two Factors in Visual Fatigue Caused by Stereoscopic HDTV Images," Displays, 25, 141-150, 2004.

[74] F. Speranza, W. J. Tam, R. Renaud, N. Hur, "Effect of Disparity and Motion on Visual Comfort of Stereoscopic Images," Proc SPIE, 6055, 94-103, 2006.

[75] M. Emoto, K. Masaoka, Y. Yamanoue, M. Sugawara, Y. Nojiri, "Horizontal Binocular Disparities and Visual Fatigue while Viewing Stereoscopic Display," VISION, 17(2), 101-112, 2005.

[76] K. Ukai, P. A. Howarth, "Visual Fatigue Caused by Viewing Stereoscopic Motion Images: Background, Theories and Observations," Displays, 29, 106-116, 2008.

[77] M. Lambooji, W. Ijsselstejin, M. Fortuin, I. Heynderickx, "Visual Discomfort and Visual Fatigue of Stereoscopic Displays: A Review," Journal of Imaging Science and Technology, 53(3), 1-14, 2009.

[78] H. Takada, M. Miyao, F. Sina, Ed, Stereopsis and Hygiene, Singapore: Springer, 2019.

[79] S. Yano, "Size of Disparity for Binocular Fusion - A Study on Stimulus Target Properties -," The transactions of the Institute of Electronics, Information and Communication Engineers, 75(10), 1720-1728, 1991.

[80] M. Emoto, S. Yano, S. Nagata, "Distribution of Fusional Vergence Limit in Viewing Stereoscopic Image Systems," The Journal of the Institute of Image Information and Television Engineers, 55(5), 703-710, 2001.

[81] Safety Guidelines Sub-Committee of 3D Consortium. 3DC Safety Guidelines. 2011.

[82] T. Kojima, "Study of Visibility and Biomedical Effects of 3D Images," Doctoral Dissertation of Graduate School of Information Science, Nagoya University, 2014

[83] T. Shiomi, K. Uemoto, T. Kojima, S. Sano, H. Ishio, H. Takada, M. Omori, T. Watanabe, M. Miyao, "Simultaneous measurement of lens accommodation and convergence in natural and artificial $3 \mathrm{D}$ vision," Journal of the Society for Information Display, 21(3), 120-128, 2013.

[84] H. Takada, K. Yokoyama, Ed, Bio-information for Hygiene, Singapore: Springer, 2021

[85] J. Suzuki, T. Matsunaga, K. Tokumatsu, K. Taguchi, Y. Watanabe, "Q\&A on Stabilometry GuideBook (1995)," Equil Res, 55, 64-77, 1996.

[86] T. Okawa, T. Tokita, Y. Shibata, T. Ogawa, H. Miyata, "Stabilometry: significance of locus length per unit area (L/A) in patients with equilibrium disturbances," Equil Res, 54, 283-293, 1995.

[87] H. Takada, Y. Kitaoka, Y. Shimizu, "Mathematical Index and Model in Stabirometly," Forma, 16(1), 17-46, 2001.

[88] H. Takada, Y. Kitaoka, M. Ichikawa, M. Miyao, "Physical meaning of geometrical index for stabilometry," Equil Res, 62(3), 168-80, 2003.

[89] M. Malińska, K. Zużewicz, J. Bugajska, A. Grabowski, "Heart rate variability (HRV) during virtual reality immersion,” Int J Occup Saf Ergon, 21(1), 47-54, 2015.

[90] A. M. Baranowski, K. Keller, J. Neumann, H. Hecht, "Genre-dependent effects of 3D film on presence, motion sickness, and protagonist perception," Displays, 44, 53-59, 2016.

[91] T. H. Cho, C. Y. Chen, P. J. Wu, K. S. Chen, L. T. Yin, “The comparison of accommodative response and ocular movements in viewing $3 \mathrm{D}$ and $2 \mathrm{D}$ displays," Displays, 49, 59-64, 2017.

[92] A. Einstein, "Über die von der molekularkinetischen Theorie der Wärme geforderte Bewegung von in ruhenden Flüssigkeiten suspendierten Teilchen," Annalen der Physik, 322(8), 549-560, 1905.

[93] A. Einstein, "Zur Theorie der Brownschen Bewegung," Annalen der Physik, 324(2), 371-381, 1905 . 
[94] M. Takada, Y. Fukui, Y. Matsuura, M. Sato, H. Takada, "Peripheral viewing during exposure to a 2D/3D video clip: effects on the human body," Environ Health Prev Med, 20(2), 79-89, 2015.

[95] P. A. Goldie, T. M. Bach, O. M. Evans, "Force platform measures for evaluating postural control: reliability and validity," Arch Phys Med Rehabil, 70, 510-517, 1989.

[96] H. Takada, Y. Shimizu, Y. Matsuura, T. Shiomi, M. Miyao, "Non-linear analysis of stabilograms with alcoholic intake," in Annu Int Conf IEEE Eng Med Biol Soc 2012, 4208-4211, 2012.

[97] K. Yoshikawa, H. Takada, M. Miyao, "Effect of Display Size on Body Sway in Seated Posture While Viewing an Hour-Long Stereoscopic Film," in Universal Access in Human-Computer Interaction. User and Context Diversity 2013 Part II, C. Stephanidis, M. Antona, Ed, Heidelberg: SpringerVerlag, 336-341, 2013.

[98] K. Fujikake, M. Miyao, R. Honda, M. Omori, Y. Matsuura, H. Takada, "Evaluation of High-Quality LCDs Displaying Moving Pictures, on the Basis of the Form Obtained from Statokinesigrams," Forma, 22(2), 199-206, 2007.

[99] K. Fujikake, H. Takada, M. Omori, M. Miyao, "Evaluation of High-Quality LCDs Displaying Moving Pictures by Use of the Form Obtained from Statokinesigrams and the Dynamics," Forma, 22(3), 217-229, 2007.

[100] R. S. Kennedy, L. E. Lane, K. S. Berbaum, M. G. Lilienthal, "A simulator sickness questionnaire (SSQ): A new method for quantifying simulator sickness," International J Aviation Psychology, 3, 203-220, 1993.

[101] S. R. Holomes, M. J. Griffin, "Correlation Between Heart Rate and the Severity of Motion Sickness Caused by Optokinetic Stimulation," J Psychophysiology, 15, 35-42, 2001.

[102] N. Himi, T. Koga, E. Nakamura, M. Kobashi, M. Yamane, K. Tsujioka, "Differences in autonomic responses between subjects with and without nausea while watching an irregularly oscillating video," Autonomic Neuroscience: Basic and Clinical, 116, 46-53, 2004.

[103] Y. Yokota, M. Aoki, K. Mizuta, Y. Ito, N. Isu, "Motion sickness susceptibility associated with visually induced postural instability and cardiac autonomic responses in healthy subjects," Acta Otolaryngologia, 125, 280-285, 2005.

[104] Y. Matsuura, H. Kato, Y. Mori, F. Kinoshita, T. Takaishi, H. Takada, "Evaluation of An Hour-Long Stereoscopic Film on Human Body by using Functional Test of Autonomic Nervous System," Bulletin of Society for Science on Form, 30(1), 66, 2015.

[105] L. M. Scibora, S. Villard, B. Bardy, T. A. Stoffregen, Wider stance reduces body sway and motion sickness," in Proceedings of VIMS 2007, 18-23, 2007.

[106] H. Takada, Y. Mori, T. Miyakoshi, "Effect of Background Viewing on Equilibrium Systems," in Universal Access in Human-Computer Interaction. Access to Interaction, M. Antona, C. Stephanidis, Ed, Heidelberg: SpringerVerlag, 2015, 255-263.

[107] K. Yoshikawa, F. Kinoshita, K. Miyashita, A. Sugiura, T. Kojima, H. Takada, M. Miyao, "Effects of Two-Minute Stereoscopic Viewing on Human Balance Function," in Universal Access in Human-Computer Interaction. Access to Interaction, M. Antona, C. Stephanidis, Ed, Heidelberg: Springer-Verlag, 2015, 297-304.

[108] F. Kinoshita, Y. Mori, M. Miyao, H. Takada, "On mathematical models of two-minute stereoscopic viewing on human balance function," Forma, 32(S), 11-17, 2017.

[109] List of Officials of Commerce and Information Policy Bureau, Ministry of Economy, Trade and Industry of Japan, Digital Content White Paper 2017, Tokyo: Digital Content Association of Japan, 2017.

[110] M. H. Fischer, "Optokinetic ausgeloste Bewegungs-wahrnehmungen und optokinetinetisher Nystagmus," Journal of Psychological Neurology, 41, 273-308, 1930

[111] T. Brandt, "Differential effects of central versus peripheral vision on egocentric and exocentric motion perception," Experimental Brain Research, 5, 476-491, 1973.

[112] A. Koohestani, D. Nahavandi, H. Asadi, P. M. Kebria, A. Khosravi, R. Alizadehsani, S. Nahavandi, "A Knowledge Discovery in Motion Sickness: A Comprehensive Literature Review," IEEE Access, 7, 85755-85770, 2019. 